\section{Perception, recognition and structuring of emotions through emojis.} signals to provide information, regulate interaction and express intimacy (Van Kleef, 2016). Researchers have found that emojis regulate the interactions of digital social media, especially in terms of expression of emotions and emotional states (Derks et al. 2008; Riva, 2002). According to other studies, when looking at emojis and real human faces, similar brain regions are activated, and hence emojis are used as facial non-verbal signs in written communication in the same way that real facial expressions are used and perceived in real communication (Churches et al., 2014). Due to the frequent use of emojis and their intensive use with each passing year, we assume that emojis can be used as an innovative method for studying the grouping of emotions in humans.

Methods. Stimuli - 33 selected emojis.

Procedure. 50 participants, aged 20-22 years, were instructed to sort emojis by their similarity in emotion. They also had the opportunity to create an unlimited number of groups for indefinite time. All participants managed to sort emojis by emotion. Their responses were then initially processed by using a $33 \times 33$ symmetric matrix, and then a cluster analysis was performed

Results

Frequency analysis. Using frequency analysis, we found, that the subjects most often use emojis that symbolize positive emotions and, much less often, emojis with negative emotional or emotionally nonspecific function (Fig.1)

\section{Background.}

Facial expressions are used as non-verbal
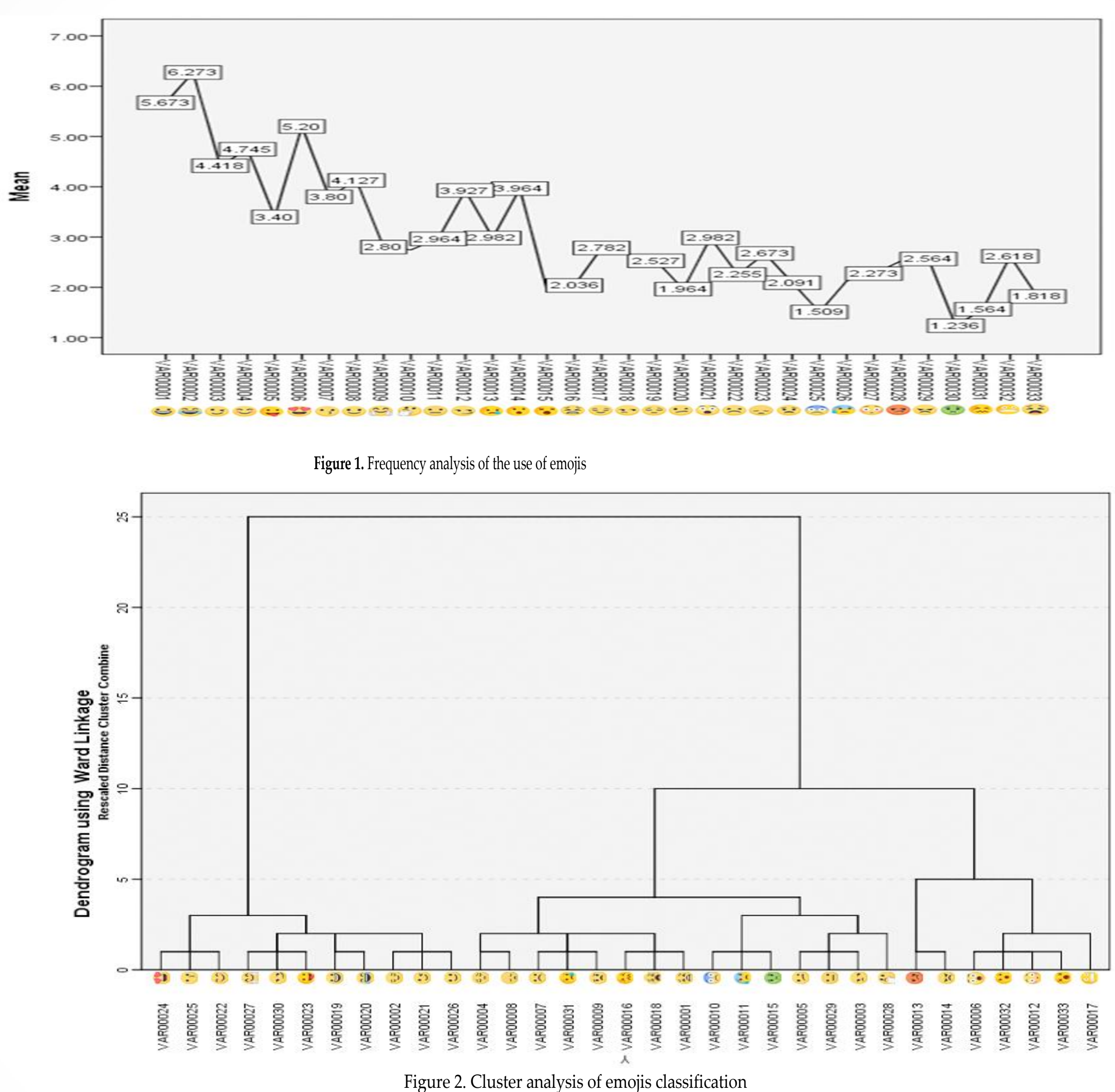

The most used emoji by female participants is Hugging Face $\frown \mathrm{F}(77.103)=4.865 ; \mathrm{p}=0.032$

Male participants use the most common Winking Face, $\because$ F $(447,657)=3,069 ; \mathrm{p}=$ 0.053)

Cluster analysis. Using a cluster analysis approach, we checked how participants grouped emojis by category in hierarchical tree clusters (Fig.2)

The first big cluster "Positive emotions“ includes emojis with positive expression, who express love; emotionally funny characters; cheerful emojis; and emojis expressing relief. The participants make a clear differentiation between joy and love emojis.

In the second cluster "Negative and Undefined emotions" negative emotions contain emojis that express negative emotions. It is subdivided into two smaller clusters: one containing emojis that express grief and disappointment; these emojis are arranged in an intense character: emojis depicting mild, moderate, and severe disappointment. In the other subcluster of the "negative emojis" there are emojis that symbolize fear / anxiety and emojis that have more neutral character.

Undefined emotions include emojis expressing negative emotions such as anger and unspecified emotions like astonishment. Surprise is a prerequisite most often for situations that cause negative emotions as anger rather than positive emotions.

\section{Discussion}

gure 2. Cluster analysis of emojis classification

Wood, Duder S. conducted an experiment and found that some emojis are actually used ambiguously and interpreted differently, but they are still reliable indicators of emotional states. Assuming that emotion structure as emotional expression is sufficiently adequate and reliable for the intranslation of emotional states, our research does not confirm the results of Jack et al. (2014) according to which anger and disgust, and also surprise and fear are similar emotions, because emojis expressing these emotions fall into different clusters, that is, they are perceived as very different emotions.

References

Van Kleef, G A. The interpersonal dynamics of emotion: Cambridge University Press,2016.

Derks, D., Bos, A.E., \& Grumbkow, J.V. Emoticons in computer-mediated communication: social motives and social context. Cyberpsychology \& behavior 2008; 11(1), 99-101. doi: 10.1089/cpb.2007 9926 Riva, G. The sociocognitive psychology of computer-mediated communication: The present and future of technology-based interactions. CyberPsychology \& Behavior, 2002, 5, 581-598. doi:10.1089/109493102321018222 Riva, G. The sociocognitive psychology of computer-mediated communication: The present and future of technology-based interactions. CyberPsychology \& Behavior, 2002, 5, 581-598. doi:1010
Churches, O., Nicholls, M., Thiessen, M., Kohler, M., \& Keage, H. Emoticons in mind: An event-related potential study. Social Neuroscience, 2014, 9, 196-202. doi:10.1080/17470919.2013.873737 